\title{
Probing the evolution of magnetic fields in clusters and galaxies
}

\author{
Paul Alexander', Martin Krause, Rosie Bolton, Julia Riley, Joern Geisbuesch \\ Astrophysics Group, Cavendish Laboratory, J.J. Thomson Avenue, Cambridge, CB3 OHE, U.K. \\ E-mail: Paul.Alexanderemrao.cam.ac.uk
}

Probing the structure and evolution of magnetic fields in clusters, especially with cosmological epoch, will be a major tool in understanding the role of magnetic fields in structure formation and also the origin of cosmic magnetism. In this paper we investigate the ability of the SKA and its pathfinders to probe magnetic fields in clusters out to moderately high redshift via observations of background polarised synchrotron sources. We develop models for the distribution of polarised sources and also for the distribution and field structure in clusters. Various possible experiments are then considered which show that the SKA pathfinder instruments, Phase I SKA and the full SKA provide an excellent set of tools for probing the structure and cosmological evolution of magnetic fields in clusters.

From planets to dark energy: the modern radio universe University of Manchester, Manchester, UK

1-5 October, 2007

\footnotetext{
$1 \quad$ Speaker
} 


\section{Introduction}

Probing the structure and evolution of magnetic fields in clusters, especially with cosmological epoch, will be a major tool in understanding the role of magnetic fields in structure formation and also the origin of cosmic magnetism. One can envisage two extreme models for the origin of magnetic fields in clusters. The first is that the fields are injected into the cluster gas, either via galactic winds are AGN ejection. Secondly the fields could be present in the primordial baryonic gas as it in falls into the forming cluster. Quite possibly both occur, but in event fluid dynamical effects within the cluster itself will modify the field, for example the amplification of field by a turbulent dynamo. A powerful tool to probe these processes is to observe Faraday rotation (and depolarisation) against either embedded or background polarised synchrotron sources.

There is a large body of work which has attempted to probe fields in nearby clusters. Cluster-wide magnetic fields have been established beyond any doubt. The first successful demonstration of Faraday rotation of background sources seen through a cluster was by Vallee et al [1] in A2319. More recently, the existence of magnetic fields in the outer parts of clusters has been demonstrated by stacking Faraday rotation results from 16 Abell clusters [2]. Rotation measure also shows a strong dependence on cooling flow rate [3]. Studies of rotation against background sources are limited by the sensitivity to detect a sufficiently large number of polarised background sources - this situation will be greatly improved by the next generation of radio interferometers especially the SKA as we discuss below. Observations of rotation against embedded radio sources have produced very good data, but usually over only a fraction of a cluster - the technique is also clearly biased towards those clusters containing active radio AGN. The results of for example Murgia et al. [4] show evidence for a turbulent, or at least random, magnetic field structure. A comprehensive discussion of the literature is given in [5].

In this paper we investigate the likely ability of the SKA and its pathfinders to probe magnetic fields in clusters out to moderately high redshift via observations of background polarised synchrotron sources. We develop models for the distribution of polarised sources and also for the distribution and field structure in clusters. Various possible experiments are then considered which show that the SKA pathfinder instruments, Phase I SKA and the full SKA provide an excellent set of tools for probing these problems.

\section{Simulating the Faraday Screen and the Rotation Measure Grid}

For large sky area simulations we construct a cluster model for the distribution and masses of clusters including their evolution with cosmic epoch based on a Jenkins mass function. Each cluster is then assigned an electron distribution following a standard $\beta$-profile with a mean core radius of $100 \mathrm{kpc}$. Rotation Measure (RM) distributions with radius in the cluster are assigned based on observations of clusters in the local universe. We then construct an RM image of the sky (see Figure 1). 
The sources which will form the RM grid are determined from the total intensity luminosity function models developed as part of the European SKA Design Study (SKADS) consortium. These models include populations of radio loud AGN and star-forming galaxies. Each source is assigned a fractional polarization as a function of frequency and total intensity. The procedure is based on the models of [6] and uses a log-normal distribution at all frequencies. Typical polarisations are of order $1 \%$ and no evolution of the polarisation fraction is used. Spacing of sources on the RM grid is very similar to other estimates. Importantly however we are able to determine the occurrence of background sources behind clusters out to high redshifts

\section{Imaging the RM sky with the SKA}

We illustrate the ability of the SKA to image the RM sky in Figure 1. Here we show a model image of the RM sky including the contribution from all clusters and groups. We also show images of the line-of-sight RMs recovered against all the sources which could be detected with a typical SKA sensitivity. When slightly smoothed we see that we image essentially the complete RM sky. This simulation does not include any other foregrounds; although the sky is well measured using this information to infer the evolution of fields in clusters (and galaxies) requires considerable analysis (see Geisbuesch \& Alexander this volume). We consider this briefly in the next section for some experiments which can be performed with pathfinder telescopes. Further this image is at a rest-frame of $1.4 \mathrm{GHz}$. We also discuss below (see also Krause et al. this volume) how the turbulent structure of magnetic fields in clusters will lead to depolarisation at lower frequencies and provide an additional probe of field structure on size scales smaller than a beam.
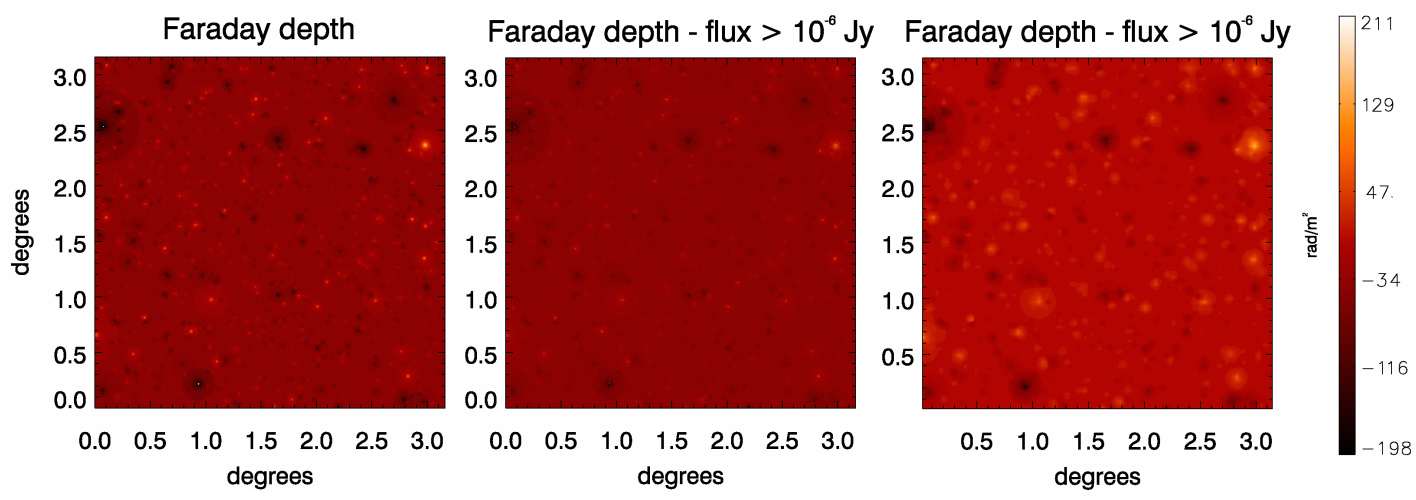

Figure 1: Simulated Faraday depth image of the sky from clusters and groups. The left panel shows the sky simulation. The middle panel shows the recovered RM sky against background sources with flux densities greater than $1 \mathrm{mJy}$ and the right panel shows the same results slightly smoothed. 


\section{Experiments with SKA pathfinders}

So as to consider possible experiments with SKA pathfinder telescopes and the 10\% SKA we use a definition of three virtual telescopes. These virtual telescopes have been introduced for the science simulation effort within SKADS and the basic parameters are discussed in the next table. The SVT-2 can be considered a hybrid of the MeerKAT and ASKAP pathfinders; SVT 3 is an approximation to one version of Phase 1 of the SKA.

\begin{tabular}{|l|l|l|l|}
\hline & SVT 2 & SVT 3 & SVT 3 AA \\
\hline $\mathrm{T}_{\text {sys }}$ & $30 \mathrm{~K}$ & $30 \mathrm{~K}$ & $50 \mathrm{~K}$ \\
\hline Collecting Area & $14,000 \mathrm{~m}^{2}$ & $56,000 \mathrm{~m}^{2}$ & $20,000 \mathrm{~m}^{2}$ \\
& & & \\
\hline FoV / deg & & $4(1.4 / \mathrm{f})^{2}$ & 250 \\
& $4(1.4 / \mathrm{f})^{2}$ & & \\
\hline
\end{tabular}

In Figure 2 we show histograms which give the number of clusters detected with the specified number of background polarised sources for $1 \mathrm{hr}$ and $100 \mathrm{hr}$ integrations for each of the telescopes. It is clear that even pathfinder instruments will be an excellent tool for establishing an RM grid.
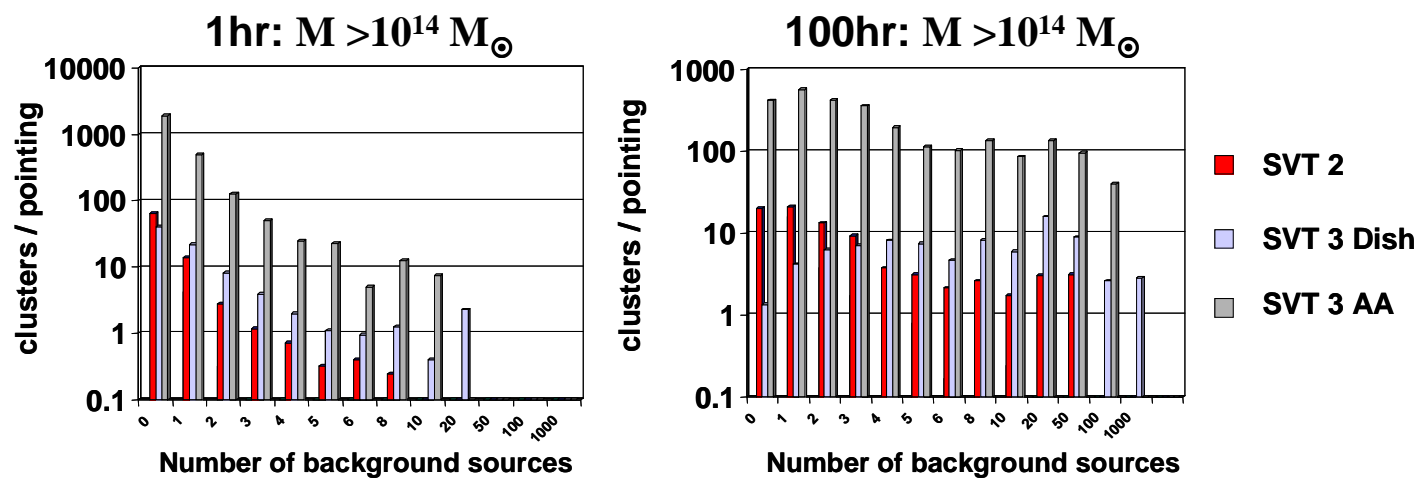

Figure 2: Histograms showing the number of clusters detected with the given number of background sources for observations of $1 \mathrm{hr}$ and $100 \mathrm{hr}$ with each virtual telescope. Going a factor of three lower in cluster mass, increases the numbers by about an order of magnitude in all bins.

We now consider some specific experiments and follow a normal wedding-cake approach. The first experiment is a wide-field shallow survey of 100 square degrees taking 1000 hours for an instrument such as MeerKAT or $\sim 200$ hours with ASKAP. We assume that all clusters will have X-ray of SZ measurements and we know to sufficient accuracy the redshifts of the clusters and background sources (estimated photometrically with sufficient accuracy using e.g. SDSS). Such a survey would detect more than 100 clusters with more than 20 background sources and more than 1000 clusters with masses greater than $10^{14} \mathrm{M}_{\odot}$ with at least one line-of- 
sight to a background source. Such a survey will be ideal to form a census of the field strengths and also the magnetic-field structure in clusters in the nearby universe.

The second experiment is a $\sim 100 \mathrm{~h}$ integration (with an instrument such as MeerKAT) on a single deep field. This will yield $\sim 19$ clusters in the redshift range 0.2 to $0.5,24$ clusters between redshift 0.5 and 1 and 10 clusters at redshifts greater than 1 . Observing say six well-studied fields (e.g. GOODS, XMM-LSS-DXS, VISTA-Video1, DEEP2, COSMOS, Vimos4-DXS) will enable to do a preliminary study of the evolution of magnetic fields out to high redshift.

Parameterising the cosmological evolution of the field strength in clusters as $(1+z)^{\alpha}$, our results suggest that in $\sim 600 \mathrm{hr}$ of observing should be able to constrain $\alpha$ to about 0.3 .

The third type of experiment would be targeted deep surveys of nearby clusters for which there was specific scientific interest (see Figure 4) . One possibility is to examine the strength of the magnetic field close to cold fronts the existence of which require the suppression of thermal conductivity by several orders of magnitude relative to the Spitzer value - this can be done easily via magnetic fields reducing the

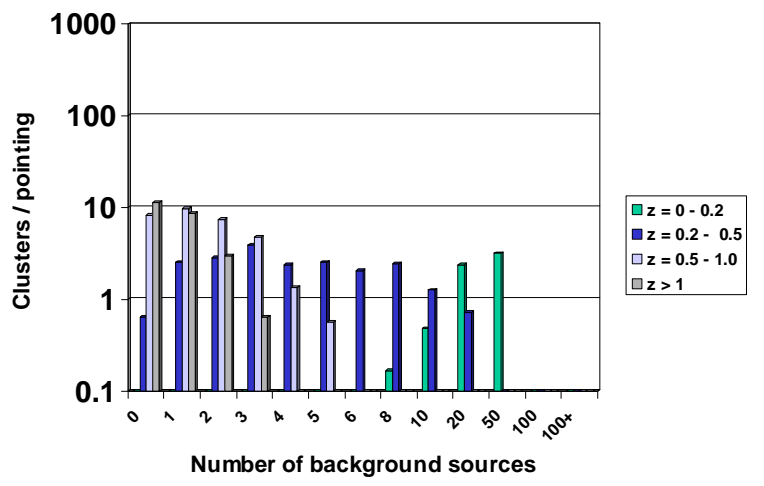

Figure 3: Distribution of clusters with the specified number of background sources as a function of redshift.

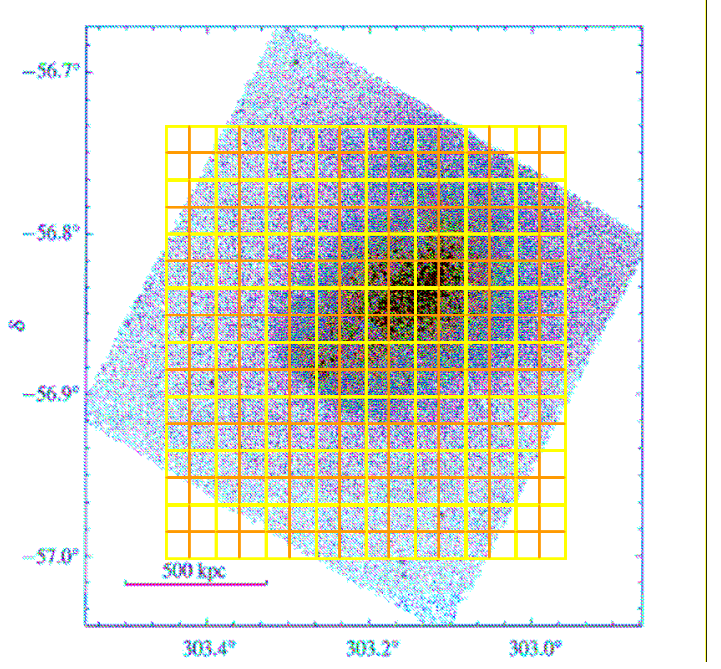

Figure 4: The cluster A3667 showing the density of the RM grid. mean free path of the thermal electrons.

\section{Random and turbulent magnetic field structures}

Observations against embedded radio sources in local clusters suggest that the field structure is likely to have a significant random structure possibly related to turbulence in the intra-cluster medium. If there is structure in the foreground RM screen on scales smaller than the resolution of the telescope beam then observations will suffer beam depolarisation. This problem is made worse if the background source has an angular size which is larger than the angular size of the structure in the foreground screen. Furthermore the behaviour is strong function of frequency. A detailed analysis of these effects is given in Krause et al. of this 
volume. In Figure 5 we show examples of models of the magnetic field component for different power-law forms for power on different spatial scales.
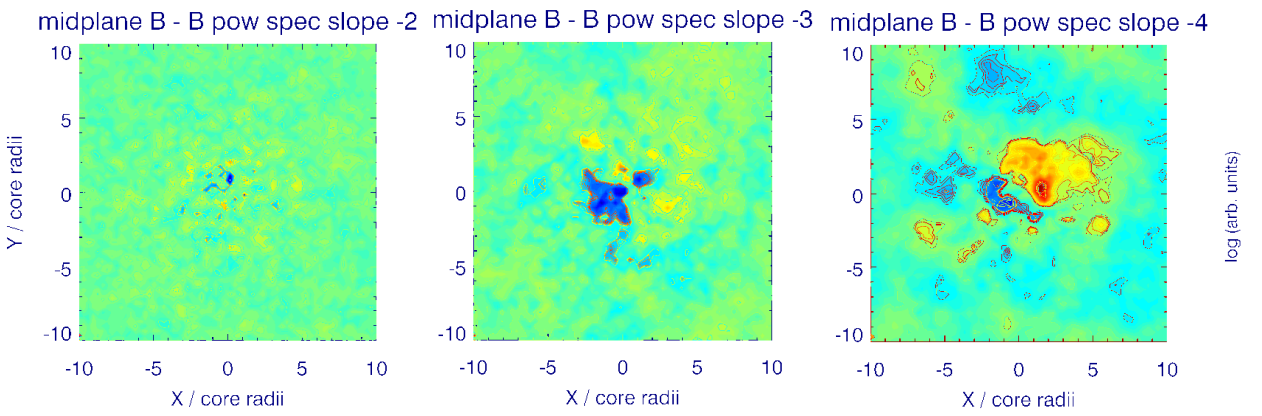

Figure 5: Examples of mid-plane magnetic field calculated for different power-law forms for the spatial power spectrum.

\section{Conclusions and further work}

The SKA and even its pathfinders will be excellent instruments to probe magnetic fields in clusters both in the local universe and out to relatively high redshifts. The modelling we have currently completed gives a reasonable representation of the RM sky and source population making up the RM grid. The next stage of the modelling will be to include better models for the polarisation structure of the (evolving) source population and also to continue to improve models for the field structure in the foreground screen.

\section{References}

[1] J.P. Vallee, J.M. MacLeod \& N.W. Broten, A\&A 156 (386) 1986

[2] T.E. Clarke, P.P. Kronberg \& H. Böhringer, ApJ 546 (L111) 2001

[3] G.B. Taylor, S.W. Allen \& A.C. Fabian, MNRAS 334 (769) 2002

[4] M. Murgia et al., A\&A, 424 (429) 2004

[5] C.L. Carilli \& G.B. Taylor, Ann Rev Astron Astrophys 40 (319) 2002

[6] M. Tucci et al., MNRAS 349 (1267) 2004 